\title{
Aspectos socioculturales que influyen en los accidentes laborales en los trabajadores de la salud de la salud
}

\author{
Araceli Sánchez Ramos*
}

RESUMEN

Los accidentes laborales son todos aquellos estados patológicos derivados de la acción continua de una causa que tenga su origen en el trabajo o en el medio en que se desarrolla el mismo. La industrialización y el modelo económico que ha venido imperando en el mundo, ha traído aparejadas las mejoras en la producción de bienes y servicios, lo que permitió a las poblaciones un acceso más fácil a ellos, pero también trajo consecuencias sociales como la pobreza, el desempleo y la precarización del trabajo, lo que ha impactado de forma muy negativa en la salud de los trabajadores, incrementando el número de accidentados y enfermedades ocupacionales. Todo este panorama en el que se encuentran sumergidos los trabajadores de la salud, donde el desarrollo tecnológico alcanzado y las condiciones del medio ambiente, en general, han modificado su entorno laboral y, por consiguiente, su adaptación en el que los peligros o factores de riesgo en el trabajo se han transformado ocasionando agravios en su salud, se desarrollará en el presente ensayo.

Palabras clave: Aspectos culturales, accidentes laborales, trabajadores de salud.

\section{Socio-cultural aspects that influence in occupational accidents in health care workers}

\begin{abstract}
Work accidents are those pathological conditions resulting from continued action of a cause that has its origin in the work or in the environment in which it develops. Industrialization and economic model that has prevailed in the world brought about improvements in the production of goods and services that allowed people easier access to them but also brought social consequences such as poverty, unemployment, job insecurity, that has impacted very negatively on the health of workers by increasing the number of accidents and occupational diseases. This panorama which is submerged health care workers where technological development achieved and the conditions of the general environment have modified their work environment and, therefore, its adaptation where hazards or risk factors in the work have transformed causing health grievances will be developed in this essay.
\end{abstract}

Key words: Socio cultural aspects, occupational accidents, health care workers.

* Instituto Nacional de Neurología y Neurocirugía "Manuel Velasco Suárez”.

Correspondencia: MEE. Araceli Sánchez Ramos.

E-mail: sanchez-araceli1@hotmail.com

Este artículo puede ser consultado en versión completa en http://www.medigraphic.com/enfermerianeurologica 


\section{INTRODUCCIÓN}

$\mathrm{L}$ os accidentes laborales son todos aquellos estados patológicos derivados de la acción continua de una causa que tenga su origen en el trabajo o en el medio en que se desarrolla este mismo.

La industrialización y el modelo económico que ha venido imperando en el mundo ha traído aparejadas las mejoras en la producción de bienes y servicios, lo que ha permitido a las poblaciones un acceso más fácil a ellos, pero, también trajo consecuencias sociales como la pobreza, el desempleo y la precarización del trabajo; ello ha impactado de forma muy negativa en la salud de los trabajadores, incrementando el número de accidentados y enfermedades ocupacionales. Todo este panorama en el que se encuentran sumergidos los trabajadores de la salud, donde el desarrollo tecnológico alcanzado y las condiciones del medio ambiente en general, han modificado su entorno laboral y, por consiguiente, su adaptación donde los peligros o factores de riesgo en el trabajo se han transformado ocasionando agravios en su salud, se desarrollará en el presente ensayo.

\section{DESARROLLO}

El accidente de trabajo se define legalmente, como "aquel que ocurre por el ejercicio del trabajo a servicio de la empresa, provocando una lesión corporal o una perturbación funcional causante de muerte, pérdida o reducción permanente o temporaria de la capacidad de trabajo".

En las últimas décadas ha habido importantes avances tecnológicos en los diferentes lugares de trabajo, los cuales, junto con los factores socioculturales han transformado el trabajo para muchas personas en todo el mundo. Los efectos de dichos cambios en la seguridad y la salud en el trabajo (SST) también han sido notorios. En algunos casos, se han reducido o eliminado los peligros y riesgos más tradicionales, por ejemplo, a través de la automatización industrial, pero las nuevas tecnologías también han creado nuevos riesgos. Muchos de los riesgos tradicionales del lugar de trabajo, sin embargo, persisten y el número de enfermedades y accidentes relacionados con el trabajo sigue siendo elevado. Al mismo tiempo, muchos trabajadores están expuestos a "nuevos" riesgos generados por las características cambiantes del trabajo, por ejemplo, debido a las condiciones derivadas del empleo precario y a la creciente presión para satisfacer las exigencias de la vida laboral moderna. Los perfiles de edad de la fuerza de trabajo también están cambiando, al igual que el equilibrio de género en muchos lugares de trabajo. Estos cambios en las características del empleo han creado riesgos manifiestos que anteriormente eran menos destacados o menos evidentes. ${ }^{2}$
Los trabajadores de la salud constituyen un colectivo particular de trabajadores expuestos a riesgos específicos de su trabajo. El impacto de los riesgos presentes en su lugar de trabajo sobre la salud se sabe que es alto, aunque debido al subregistro, éste es de difícil cuantificación.

Si bien los accidentes de trabajo de tipo punzocortante con riesgo biológico (Hepatitis, VIH, etcétera) aparecen como los más comunes, no son la única manera por la cual los trabajadores de la salud puedan perder su salud.

Un accidente de trabajo es siempre el resultado de la interacción de múltiples factores entre los que se destacan los propios del medio ambiente de trabajo (condiciones físicas ambientales de trabajo, equipos de trabajo, organización de trabajo, ritmos de trabajo, relaciones de trabajo, etcétera), y los del individuo (características antropológicas, carga, fatiga, calificación, nutrición, estado de salud, etcétera). ${ }^{3}$

Por tanto, todo este panorama en el que se encuentra sumergido el trabajador de la salud y en el que el desarrollo tecnológico alcanzado y las condiciones del medio ambiente en general han modificado su entorno laboral y, por consiguiente, su adaptación, además en donde los peligros o factores de riesgo en el trabajo se han transformado y han surgido otras enfermedades y en el que la salud del trabajador se halla constantemente amenazada, ya no sólo por los problemas o situaciones de la vida laboral, sino por todas las condiciones a que en la vida familiar y extralaboral, nos lleva los factores socioculturales.

Es por esto que con el presente ensayo se pretende destacar como los factores socioculturales influyen en los accidentes laborales de los trabajadores de la salud. Para ello, se pretende abordar los mismos, primero, explicando sobre los cambios de las condiciones de vida y salud de los trabajadores que ocasionó la revolución industrial, hasta llegar a la globalización actual que sufre el mundo; además, como la sociología médica establece una relación entre la enfermedad vista como un fenómeno social y el modo de producción capitalista que genera las condiciones adversas para la salud de los trabajadores es importante también abordar el habitus, mencionando cómo las actitudes y conductas definen lo saludable o nocivo que puede ser el estilo de vida de los trabajadores de la salud y finalmente, cómo los trabajadores de la salud pueden evitar estos accidentes para aspirar a una calidad de vida.

La revolución industrial ocasionó cambios en las condiciones de vida y salud de los trabajadores, razón por la cual diversos investigadores, identificados con la causa de los trabajadores, comenzaron a estudiar el impacto de ésta sobre la salud de la población trabajadora. Marx y Engels dedicaron gran parte de su obra a analizar los efectos sobre la salud de los trabajadores como una de las expresiones concretas de la explotación capitalista. ${ }^{4}$ 
Las consecuencias del desarrollo capitalista se manifiestan en forma concreta en la salud de los grupos sociales que componen las distintas sociedades históricamente determinadas: surgen nuevas enfermedades o se acentúa la presencia de otras y se intensifican los accidentes. El surgimiento y las características de la enfermedad se presentan, por tanto, como resultado de la forma en que la sociedad produce y se reproduce, lo que responde a las características que el capitalismo impone en la dialéctica de su desarrollo. ${ }^{5}$

Las repercusiones de la revolución industrial, específicamente en el área de la salud, se reflejan en que los trabajadores presentan problemas frecuentes de salud física y mental, aparentemente relacionados al trabajo y a los variados factores de riesgos ocupacionales existentes en los ambientes laborales. Los problemas mencionados evidencian la presencia de cansancio físico y mental, la pérdida de sueño y de apetito, la necesidad de utilizar ansiolíticos y/u otras drogas, adelgazamientos y/o aumento de peso corporal, episodios de llanto, sensación de tristeza, cefaleas, dolores articulares y otros, aparentemente advenidos del trabajo o su exceso.

La serie de agravios a la salud presentada por los trabajadores del área de la salud, durante el transcurrir de los años se ha intensificado.

Los cambios generados por la industrialización y por la apertura económica influenciaron a las personas, la sociedad y las organizaciones que necesitaron transformar sus estructuras, actividades y procesos para seguir siendo competitivos. Sin embargo, las modificaciones en la sociedad y en las relaciones de producción fueron acompañadas de transformaciones en las manifestaciones de sufrimiento y psicopatología de los individuos. En el área de la salud, trabajar durante muchas horas en ambientes laborales insalubres puede representar riesgos a la salud de los trabajadores. Entre las mujeres trabajadoras existe una interacción entre factores estresantes del trabajo y extra laborales, porque se asocian las demandas de trabajo con las demandas sociales y familiares. ${ }^{6}$

Por tanto, las consecuencias sociales de este modelo económico de desarrollo además de concentrar capital, producir pobreza, desempleo, flexibilización y precarización del trabajo han impactado de forma muy negativa en la salud de los trabajadores, incrementando el número de trabajadores accidentados y enfermedades ocupacionales. ${ }^{7}$

Otro aspecto importante es la globalización actual que sufre el mundo y que ha tenido también repercusión en el estado de salud de los trabajadores de los pueblos de América Latina y del Caribe. Los bajos salarios, el aumento del empleo en el sector informal, el empleo infantil, la industria maquiladora, las nuevas tecnologías, la automatización, la informática, la robotización y la microelectrónica han representado riesgos significativamente nuevos que afectan el estado de salud de los trabajadores. Estos son sólo algunos ejemplos de la influencia globalizadora en el sector laboral, pues todo esto ha llevado a un aumento de las enfermedades ocupacionales en estos países como las intoxicaciones, las alteraciones psíquicas, las enfermedades osteomioarticulares y los accidentes laborales.

Al reflexionar sobre la problemática de la accidentalidad laboral en los países en desarrollo, debemos tener presente las condiciones adversas en las que los trabajadores, en ocasiones, realizan su labor diaria; tanto desde el punto de vista ambiental, como desde el punto de vista de la tecnología anticuada-como cuando se introducen nuevas tecnologías-; las personas no está entrenadas en el funcionamiento de la misma y esto puede ser otro factor de riesgo. Asimismo el factor humano que viene dado por las tensiones laborales y extralaborales, unido al desempleo disminuye la concentración en el trabajo, lo que puede ocasionar accidentes durante el ejercicio de su práctica. También es importante tomar en cuenta las situaciones de malnutrición y enfermedades comunes que ejercen un efecto negativo sobre el trabajador. ${ }^{8}$ La globalización es una realidad; ésta ha resultado muy rentable para algunos y no cabe duda de que ha generado muchos beneficios indirectos desde el punto de vista de la transferencia del conocimiento. Sin embargo, también está teniendo repercusiones desastrosas para muchos sectores. Los trabajadores de salud (TS) trabajan ahora más que nunca y es frecuente que estén expuestos a los riesgos biológicos que están presentes en todos los lugares de trabajo del sector de salud que incluyen patógenos transmitidos por aire y sangre, tales como los agentes causales de tuberculosis, el síndrome agudo respiratorio severo (SARS), la hepatitis y la infección por VIH/SIDA. El personal de salud (PS) también está expuesto a sustancias químicas tales como los agentes desinfectantes y esterilizantes que provocan dermatitis, asma profesional y carcinomas, así como a drogas riesgosas que a la vez son toxinas para la reproducción, riesgos ergonómicos debido al peso de los y las pacientes que tienen que cargar y manejar directamente, sobreesfuerzos, falta de personal, rotación de turnos de trabajo y riesgos físicos tales como ruido y radiaciones entre otros. Además de las categorías de riesgos ocupacionales antes expuestas, el PS experimenta estrés por ser responsable directo del cuidado de varios pacientes enfermos o moribundos que sumado a una carga excesiva de trabajo, puede afectar seriamente su salud y bienestar.

A pesar de lo anteriormente mencionado el trabajo se considera primordial en el desarrollo psicológico, biológico y social del ser humano, pues representa la vía fundamental de relación y comunicación con la sociedad en la que se vive. Es conocido que existe una relación directa entre el 
medio ambiente laboral y el estado de salud, como bien señala Raúl Rojas Soriano: “dime en qué trabajas y te diré de qué te enfermas".

Por esto, la sociología médica se encarga de estudiar las causas de las enfermedades de trabajo, en la que se menciona que las consecuencias del desarrollo capitalista se manifiesta en forma concreta en la salud de los grupos sociales que componen a las distintas sociedades históricamente determinadas, por lo que surgen nuevas enfermedades o se acentúa la presencia de otras. Estos problemas de salud o accidentes laborales pueden encontrarse en las características de los materiales, las herramientas o los instrumentos que se utilizan, así como en el ritmo y el ambiente laboral y la organización. ${ }^{9}$

Los accidentes y las enfermedades que adquieren los trabajadores de la salud durante el ejercicio de su profesión no tienen como únicos responsables a los factores de riesgo directamente relacionados con el proceso de trabajo o con las condiciones del mismo, sino que el estilo de vida tiene también peso importante en la causalidad de los mismos.

Por estilo de vida, se entiende el patrón de conducta que tienen los trabajadores, el cual se define por las características de su trabajo, la alimentación, el ejercicio que realiza, las adicciones que tiene, el tiempo que descansan y duermen, el tipo de recreación que eligen y su actitud ante la prevención y la atención de su salud; estos factores están presentes de manera permanente en los trabajadores cuyas características repercuten necesariamente en beneficio de su salud o perjuicio de ésta. Como se puede observar las actitudes y conductas que se tiene ante estos aspectos definen lo saludable o nocivo que puede ser el estilo de vida.

El conocimiento y valoración en forma integral de todos sus factores permiten establecer los patrones de conducta de los trabajadores que están interactuando en forma constante con los factores de riesgo laborales. ${ }^{10}$

P. Bourdieu señala que cualquier profesión u oficio conduce a que se conforme un habitus propio de la profesión y que dicho habitus esté constituido por un conjunto de técnicas, referencias y creencias que se constituyen en estructuras y esquemas mentales que llevan a que las personas tengan determinadas prácticas que determinan los estilos de vida de los trabajadores de la salud. ${ }^{11,12}$

La salud del trabajador ocupa cada día un lugar más importante en la vida moderna; es por ello que el mayor nivel de información que se tenga sobre los riesgos a los que todo el personal está expuesto contribuirá de manera especial a la promoción y prevención de salud sobre las enfermedades y garantizar una mejor calidad de vida.

En algunos países industrializados a menudo se tratan las lesiones producidas durante la práctica profesional de las personas. Ahora bien, importa recordar que no es lo mismo tratar un problema que evitarlo antes de que ocurra. La prevención debe ser el primer objetivo en la presencia de accidentes laborales.

La aplicación de medidas preventivas en los lugares de trabajo reporta muchos beneficios evidentes para el trabajador, además de unas condiciones laborales más sanas y seguras; para el empleador, el beneficio más patente es el aumento de la productividad. Se requiere, por tanto, que el trabajador de la salud dirija y controle su trabajo; es necesario lograr una mejor y mayor compatibilidad entre el trabajador y los medios técnicos que utiliza para realizar su labor profesional. Las lesiones causadas a los trabajadores por herramientas o puestos de trabajo mal diseñados pueden ser muy costosas, debido a los dolores y sufrimientos que causan, por no mencionar las pérdidas financieras que suponen para los trabajadores y sus familias.

Se requiere, por tanto, que en los hospitales se dirija el término calidad de vida en el trabajo (CVT). ${ }^{13}$

\section{CONCLUSIONES}

Las consecuencias de factores socioculturales como es la industrialización y el capitalismo, además de concentrar capital, producir pobreza, desempleo, flexibilización y precarización del trabajo ocasiona cambios de las condiciones de vida de los trabajadores además de que impacta de forma muy negativa en su salud, incrementando el número accidentes y enfermedades ocupacionales.

Específicamente en el área de la salud, los avances tecnológicos junto con la rápida globalización han transformado el trabajo para muchas personas en todo el mundo, por lo que es un hecho que los trabajadores presenten accidentes laborales. Desde el punto de vista de la sociología médica, se establece una relación entre la enfermedad vista como un fenómeno social y el modo de producción capitalista; pero también tienen que ver las actitudes y conductas que definen lo saludable o nocivo que puede ser el estilo de vida de los trabajadores de la salud para que se pueda presentar un accidente laboral. Se requiere que el trabajador de la salud aplique medidas preventivas en su lugar de trabajo para garantizar una mejor calidad de vida.

\section{BIBLIOGRAFÍA}

1. Nieto H. Salud Laboral. Disponible en: http://www.fmed.uba.ar/depto/ sal_seg/salud_laborall.pd

2. Organización Internacional del Trabajo. Riesgos emergentes y nuevos modelos de prevención en un mundo de trabajo en transformación [Internet]. Ginebra: Organización Internacional del Trabajo; 2010. Disponible en: http://www.ilo.org/wcmsp5/groups/public/---ed_protect/---protrav/--safework/documents/publication/wcms_124341.pdf

3. Saliba Garbin CA, Graca A, Reis R, Elaine P. Accidentes de trabajo que afecta a los trabajadores de la salud [Internet]. Acta Odontológica Vene- 
zolana. 2009; 47 (1): 1-9. Disponible en: http://www.actaodontologica. com/ediciones/2009/1/accidentes_trabajo_profesionales_salud.asp

4. Gabaldón A. Relación salud-trabajo y desarrollo social: visión particular en los trabajadores de la educación [internet]. Rev Cubana Salud Pública. 2006; 32 (1). Disponible en http://bvs.sld.cu/revistas/spu/vol32_1_06/ spu12106.htm

5. Rojas S. Sociología médica. 3a edición. México: Plaza y Valdés Editores; 1988: p. 20.

6. Robazzi ML, Chávez MY, Barcellos R, Almeida L, Oliveira I, Pedrao LJ Exceso de trabajo y agravios mentales a los trabajadores de la salud [Internet]. Disponible en: http://bvs.sld.cu/revistas/enf/vol26_1_10/enf09110.htm

7. Feo O. Globalización y salud de los trabajadores. Salud trabajadores. 2002; 10 (1-2): 1-11.

8. Pintos Valluerca $\mathbf{J}$ et al. Influencia del Neoliberalismo y la Globalización en el estado de salud de los trabajadores de América Latina y el Caribe (Parte I) [internet]. [acceso 15 de noviembre 2011]. Disponible en: http://www.ilustrados.com/tema/7911/Influencia-NeoliberalismoGlobalizacion-estado-salud-trabajadores.html/

9. Rojas S. Op cit. p. 20

10. Macedo L, Nava R, Valdés B. El estilo de vida, factor de riesgo en la causalidad de las enfermedades y accidentes de trabajo [internet]. Revista Latinoamérica de la salud en el trabajo. 2001; 1: 93-95. Disponible en: http://www.bvsde.paho.org/bvsacd/cd46/estilo.pdf

11. Bourdieu P. Cuestiones de sociología. Madrid: Itsmo; 2000: 75-118 Disponible en: http://www.scielosp.org/scielo.php?script=sci_nlinks\& ref $=3025517 \&$ pid $=$ S0036-363420040004000070000

12. Geertz C. Conocimiento local. Ensayos sobre la interpretación de las culturas. Barcelona: Paidós; 1994: 182.

13. García L, Valdés M, Laffita A. Enfermedades profesionales en los estomatólogos, propuesta de ejercicios físicos para su prevención. Disponible en: http://www.revistaciencias.com/publicaciones/EkplVykkVZGygRkPJQ.php Fecha de consulta 21 de noviembre de 2011 